\title{
Editorial \\ The changing landscape of the clinical value of the PM/Scl autoantibody system
}

\author{
Michael Mahler ${ }^{1 *}$ and Marvin J Fritzler ${ }^{*}$ \\ 1Dr Fooke Laboratorien GmbH, Mainstr., 41469 Neuss, Germany \\ 2Faculty of Medicine, University of Calgary, Hospital Dr NW, Calgary, Alberta, Canada T2N 4N1 \\ *Both authors contributed equally
}

Corresponding author: Marvin J Fritzler, fritzler@ucalgary.ca.

Published: 26 March 2009

This article is online at http://arthritis-research.com/content/11/2/106

(c) 2009 BioMed Central Ltd

See related research article by Hanke et al., http://arthritis-research.com/content/11/1/R22
Arthritis Research \& Therapy 2009, 11:106 (doi:10.1186/ar2646)

\begin{abstract}
Autoantibodies to the polymyositis/scleroderma (PM/Scl) complex have been associated with systemic sclerosis and PM/Scl overlap syndrome. The report of Hanke and colleagues in a recent issue of Arthritis Research and Therapy is the first to describe the separate evaluation of anti-PM/Scl-75c and PM/Scl-100 autoantibodies and their relationship to clinical manifestations of systemic sclerosis. Several observations are of paramount interest, but are not in general agreement with earlier studies. These include the prevalence of anti-PM/Scl antibodies in systemic sclerosis, the association with certain clinical manifestations and prognosis of patients. This report will hopefully trigger systematic multi-centre studies to confirm and/or elucidate the novel line immunoassay and clinical associations.
\end{abstract}

\section{Background}

A characteristic feature of patients with systemic sclerosis (SSc; or scleroderma (Scl)) are anti-nucleolar antibodies (ANoAs), including autoantibodies (aabs) to components of the exosome, known also as the polymyositis/scleroderma (PM/Scl) complex $[1,2]$. While the majority of the anti-PM/Scl reactivity is directed against $\mathrm{PM} / \mathrm{Scl}-75 \mathrm{c}$ and $\mathrm{PM} / \mathrm{Scl}-100$, many of the other nine core exosome components are also targeted, albeit with lower frequency and apparently limited diagnostic value [2].

\section{Detection of PM/Scl antibodies}

Historically, anti-PM/Scl aabs were associated with $\mathrm{PM} / \mathrm{Scl}$ overlap syndrome and were detected by indirect immunofluorescence (IIF) on HEp-2 cells, immunodiffusion, immunoblotting (IB), and/or immunoprecipitation. The clinical usefulness of PM/Scl aabs detected by IB and IIF was limited due to weak reactivity on IB and interference of other ANoAs in IIF. More recently, recombinant proteins (primarily $\mathrm{PM} / \mathrm{Scl}-100$ ) have been used as antigen targets in immunoassays, and a peptide-based PM1-Alpha enzyme-linked immunosorbent assay (ELISA) was reported to demonstrate higher sensitivity than traditional methods for the detection of anti-PM/Scl aabs [2-4]. Line immunoassays (LIAs), the precursor of today's more sophisticated multiplex assays such as addressable laser bead assays (ALBIAs), have become a popular technique for the simultaneous detection of aabs [5]. Several LIAs for the detection of PM/Scl aabs are available, covering a variety of myositis- and/or SSc-associated autoantigens; all of them using the PM/Scl-100 antigen to detect anti-PM/Scl reactivity [2]. With an increasing number of diagnostic platforms to test anti-PM/Scl reactivity, more diligent attention needs to be given to standardizing the autoantigens used in assays and the various platforms (LIA, ELISA, ALBIA) in which they are employed [5].

\section{Major and early PM/Scl epitope}

Like many aab responses, aabs to the PM/Scl macromolecular complex likely undergo intermolecular epitope spreading. For example, Gutiérrez-Ramos and colleagues [6] recently reported a patient with high anti-PM/Scl aab titres identified by IIF and confirmed by IB (100 kDa band). Three months later, the patient's serum contained aabs to another $39 \mathrm{kDa}$ protein that probably corresponded to an aberrant $\mathrm{PM} /$ Scl-75, suggesting an epitope spreading phenomenon [6]. Similarly, immunization of rabbits with the PM1-Alpha peptide was attended by intermolecular epitope spreading to other exosome components [2].

$\mathrm{Aab}=$ autoantibody $; \mathrm{ALBIA}=$ addressable laser bead assays; $\mathrm{ANOA}=$ anti-nucleolar antibodies; $\mathrm{CK}=$ creatine kinase; $\mathrm{dSSc}=\mathrm{diffuse} \mathrm{SSc} ; \mathrm{ELISA}=$ enzyme-linked immunosorbent assay; IB = immunoblot; IIF = indirect immunofluorescence; LIA = line immunoassay; Scl = scleroderma; SSc = systemic sclerosis; $\mathrm{PM}=$ polymyositis. 


\section{Comments on the report of Hanke and colleagues}

Hanke and colleagues are the first to report the use of a novel LIA for the simultaneous but separate detection of $\mathrm{PM} / \mathrm{Scl}-75 \mathrm{c}$ and PM/Scl-100 aabs using a monocentric cohort of 280 SSc patients and various controls [1]. In their SSc cohort, the prevalence of anti-PM/Scl-75c was higher than anti-PM/Scl-100 (10.4\% versus $7.1 \%)$, a finding that is in keeping with previous reports that used recombinant fulllength antigens in an ELISA (10\% versus 2\%) [2,7].

When evaluating these aabs in the context of SSc subsets, $\mathrm{PM} / \mathrm{Scl}$ aabs were most prevalent in diffuse cutaneous SSc (dSSc) patients (19.8\%), a finding that is in contrast to most previous studies that found the highest prevalence in $\mathrm{PM} / \mathrm{Scl}$ overlap patients $[8,9]$. Of note, dSSc patients mainly showed an anti-PM/Scl-75c response, whereas most cases of overlap syndromes were characterized by reactivity to both $\mathrm{PM} / \mathrm{Scl}$ antigens. In agreement with PM1-Alpha ELISA results [2-4], but contrary to earlier studies [4,9], the prevalence of anti$\mathrm{PM} / \mathrm{Scl}$ reactivity, especially to $\mathrm{PM} / \mathrm{Scl}-75 \mathrm{c}$, was found to be higher in dSSc than in limited SSc.

\section{Clinical associations of anti-PM/Scl autoantibodies}

When Hanke and colleagues evaluated the clinical associations, they found that $\mathrm{PM} / \mathrm{Scl}-75 \mathrm{c} / 100$ aabs were associated with muscle disease (defined as elevated creatine kinase (CK) or atrophy), lung involvement (pulmonary fibrosis) and digital ulceration, but pulmonary arterial hypertension was found less frequently. These potentially interesting findings may have limitations since both elevated $\mathrm{CK}$ and muscle atrophy cannot be considered highly specific or sensitive parameters of inflammatory muscle disease (for example, myositis originally associated with the $\mathrm{PM} / \mathrm{Scl}$ aab system). Thus, it would be desirable if future studies would include a systematic approach to the detection and confirmation of myositis by electromyography and/or muscle biopsy.

$\mathrm{PM} / \mathrm{Scl}-75 \mathrm{c}$ aabs were detected more frequently in younger patients with more active disease and joint contractures, while gastrointestinal involvement was remarkably less frequent. Of particular interest, anti-PM/Scl-75c aabs were present in a serological subset of SSc patients with anti-Scl-70 aabs. Since Scl-70 (topoisomerase I) aabs are commonly seen in SSc $[8,10]$, careful consideration should be given to determining if $\mathrm{PM} / \mathrm{Scl}-75 \mathrm{c}$ aabs are an independent biomarker that provides incremental clinical value to the diagnosis and management of SSc patients.

\section{Comparison with other methods: quality of $\mathrm{PM} / \mathrm{Scl}-100$}

For reasons described above and in the closing below, the authors' conclusion that conventional anti-PM/Scl-100 assays may miss a relevant number of SSc patients that are positive for $\mathrm{PM} / \mathrm{Scl}$ aabs likely requires elucidation and further study.
For example, since highly characterized autoantigens are a key to high performance immunoassays, an apparent oversight in this study was the lack of a detailed description of the recombinant PM/Scl-100 antigens that were produced in bacteria or insect cells (for example, purity or presence of a fusion-tag). In addition, studies comparing this promising LIA to other established assays will be most helpful to laboratory directors that may consider adopting the LIA if cost-benefit considerations can be demonstrated to be advantageous.

\section{Conclusion}

The findings of Hanke and colleagues are of significant potential, high interest and putative clinical relevance, but at the same time they are in contrast to previous observations. An understanding of the comparative performance characteristics of the new LIA and multicentre systematic studies that evaluate multiple clinical associations (especially the presence or absence of myositis) are needed for a consensus of the clinical value of PM/Scl, and especially of $\mathrm{PM} / \mathrm{Scl}-75 \mathrm{c}$, aabs.

\section{Competing interests}

$\mathrm{MM}$ is employed at Dr Fooke Laboratorien $\mathrm{GmbH}$ selling the PM1-Alpha ELISA. MF receives honoraria for consulting services to ImmunoConcepts Inc. (Sacramento, CA).

\section{Acknowledgements}

We thank $M$ van Liempt for assistance with the references.

\section{References}

1. Hanke $K$, Brückner CS, Dähnrich $C$, Huscher D, Komorowski L, Meyer W, Janssen A, Backhaus M, Becker M, Kill A, Egerer K, Burmester GR, Hiepe F, Schlumberger W, Riemekasten G: Antibodies against $\mathrm{PM} / \mathrm{Scl}-\mathbf{7 5}$ and $\mathrm{PM} / \mathrm{Scl}-100$ are independent markers for different subsets of systemic sclerosis patients. Arthritis Res Ther 2009, 11:R22.

2. Mahler M, Raijmakers R: Novel aspects of autoantibodies to the $\mathrm{PM} / \mathrm{Scl}$ complex: clinical, genetic and diagnostic insights. Autoimmun Rev 2007, 6:432-437.

3. Mahler M, Raijmakers R, Dähnrich C, Blüthner M, Fritzler MJ: Clinical evaluation of autoantibodies to a novel PM/Scl peptide antigen. Arthritis Res Ther 2005, 7:R704-R713.

4. Mahler M, Fritzler MJ: PM1-Alpha ELISA: The assay of choice for the detection of anti-PM/Scl autoantibodies? Autoimmun Rev 2008, 8:373-378.

5. Fritzler MJ: Advances and applications of multiplexed diagnostic technologies in autoimmune diseases. Lupus 2006, 15: 422-427.

6. Gutierrez-Ramos R, Gonz Lez-Diaz V, Pacheco-Tovar MG, LopezLuna A, Avalos-Diaz E, Herrera-Esparza R: A dermatomyositis and scleroderma overlap syndrome with a remarkable high titer of anti-exosome antibodies. Reumatismo 2008, 60:296300.

7. Raijmakers R, Renz M, Wiemann C, Egberts WV, Seelig HP, van Venrooij WJ, Pruijn GJ: PM-Scl-75 is the main autoantigen in patients with the polymyositis/scleroderma overlap syndrome. Arthritis Rheum 2004, 50:565-569.

8. Ho KT, Reveille JD: The clinical relevance of autoantibodies in scleroderma. Arthritis Res Ther 2003, 5:80-93.

9. Steen VD: Autoantibodies in systemic sclerosis. Semin Arthritis Rheum 2005, 35:35-42.

10. Walker JG, Fritzler MJ: Update on autoantibodies in systemic sclerosis. Curr Opin Rheumatol 2007, 19:580-591. 